\title{
Measurement of the Vertebral Canal Dimensions of the Neck of the Rat With a Comparison to the Human
}

\author{
JAMIE R. FLYNN ${ }^{1}$ AND PHILIP S. BOLTON ${ }^{1,2 *}$ \\ ${ }^{1}$ School of Biomedical Science, Faculty of Health, University of Newcastle, Callaghan, \\ NSW, Australia \\ ${ }^{2}$ Hunter Medical Research Institute, New Lambton, NSW, Australia
}

\begin{abstract}
The aim of this study was to determine the dimensions of the vertebral canal in the neck of the rat, because little is known about the morphology of the rat's cervical spine. A comparison then was made to the vertebral canal in the neck of the human. In part 1 of this study, we determined the precision of three different methods to measure the vertebral canal. The error (coefficient of variation) in these methods was found to range from 1 to $8 \%$. In part 2 , we used a computer-based system to measure digital images of the vertebra and determined the anterior to posterior and the transverse vertebral canal dimensions in the neck of 19 young adult Sprague-Dawley rats. The anterior to posterior dimension of the vertebral canal was greatest at the upper cervical (C1-C2) level and progressively decreased in the more caudal segments (C3-T1). The transverse dimension was greatest at the atlas (C1) vertebra and smallest at the axis $(\mathrm{C} 2)$ vertebra with a steady increase in the transverse dimension with more caudal segments and a maximum transverse dimension at the level of the $\mathrm{C} 6$ and $\mathrm{C} 7$ vertebra. This study has demonstrated that the vertebral canal in the neck of young adult rats is similar in some regards to that of human. However, there are clear differences between the rat and human. These may be associated with differences in the morphology of the spinal cord or postural differences such as the cervicothoracic lordosis in bipeds compared with that in quadrupeds. Anat Rec, 290: 893-899, 2007. (c) 2007 Wiley-Liss, Inc.
\end{abstract}

Key words: cervical vertebra; neck; spine; morphology

\begin{abstract}
Animals are often used to model and study mechanisms underlying pathophysiology and disease. Recent studies of the vertebral column of the sheep (Wilke et al., 1997) and deer (Kumar et al., 2000) have been undertaken to validate these species as suitable models of the human vertebral column. These relatively large quadrupeds are thought to be suited for investigations involving segmental biomechanics. However, little is known about the central nervous system of these species, which limits the opportunity to concurrently examine nervous system function or responses to interventions in these species. In contrast, much is known about the central nervous system of the rat (Paxinos, 2004). The rat has been used to study nervous system responses to a range of circumstances, including biomechanical events involving the vertebral column sufficient
\end{abstract}

to cause spinal cord injury on the one hand (Grill, 2005) or a range of vertebral movements on the other (Sato and Swenson, 1984; Fiford et al., 2004; Lee et al., 2004; Bolton et al., 2006). However, the suitability of the use

\footnotetext{
Grant sponsor: Australian Spinal Research Foundation; Grant number: LG2000/06.

*Correspondence to: Philip S. Bolton, School of Biomedical Sciences, Faculty of Health, University of Newcastle.

Fax: 61-2-49217406. E-mail: philip.bolton@newcastle.edu.au

Received 6 September 2006; Accepted 28 December 2006

DOI 10.1002/ar.20523

Published online 15 May 2007 in Wiley InterScience (www. interscience.wiley.com).
} 
of the rat to model changes in the nervous system induced by displacements of the vertebral column in humans, as might occur during a whiplash event or spinal manipulative therapy for example, first requires knowledge of the similarities and differences between the human vertebral column and the rat.

There are now detailed accounts of the morphology of the cervical vertebra of several species such as the sheep, deer, and human (Panjabi et al., 1991; Wilke et al., 1997; Grave et al., 1999; Kumar et al., 2000; Tatarek, 2005) and reports concerning the vertebral kinematics of several species including rabbits, guinea pigs, cats, monkeys, and humans (Graf et al., 1995). However, there is little known about the vertebral column of the rat. Plain film radiographic images of the rat neck suggest that the rat, like the human, holds its head on an erect cervical vertebral column (Vidal et al., 1986). Data concerning the shape of the cervical vertebra and dimensions of the vertebral canal in the adult rat have been derived from digitized images of the cervical vertebra (Johnson et al., 1999; Kida et al., 1999). Johnson et al. (1999) have described the difference in shape of cervical and thoracic vertebra between rats and bats to examine the relationship between homologous and adjacent vertebra in different species. Their study was concerned with similarities and differences in vertebral body shape. It did not report data concerning the dimensions of the vertebral canal of these species. In contrast, a study by Kida et al. (1999) provides data on vertebral canal dimensions in the neck of the rat as part of a comparative study of the presacral vertebral columns of seven small mammals. However, the dimensions of the rat vertebra and vertebral canal in this study were only reported as "arbitrary" data relative to the vertebral dimensions of the other six small mammals reported in the study. Consequently, there are currently no quantitative data concerning the dimensions of the vertebral canal in the rat on which a comparison can be made with the human.

The primary aim of this study was to determine the morphological parameters of the cervical vertebral canal of the rat as a first step to examine the suitability of the rat's cervical vertebra as a model of the human cervical vertebra. In particular, to determine whether the anterior to posterior and the transverse dimensions of the vertebral canal in the neck of the rat have similar conformations to that of the human.

Previous morphological studies of postmortem cervical vertebral columns of the rat have used digitized images to obtain data concerning the vertebra (Johnson et al., 1999; Kida et al., 1999). In contrast, Vernier calipers have been used to directly measure the vertebra of humans (Tatarek, 2005). The measurement error for these devices was not reported in these studies. However, it was noted that the measurements made with the Vernier caliper were rounded to the nearest millimeter. This represents a potential measurement error of the order of $6-7 \%$ in the anterior to posterior dimension (see Table 4; Tatarek, 2005). Although it may seem logical to simply use direct measurement of a vertebra in the first instance, it should be noted that the irregular shape of the bony surfaces that form the vertebral canal may induce some variability and or error in the determination of the dimensions of the vertebral canal. Therefore, we undertook a study to determine the precision of each of three different strategies to measure the dimensions of the vertebral canal in the rat. One method involved direct measurement while the other two involved indirect measurement using different strategies of measuring image records of the vertebral canal. We then used the more precise of these strategies to determine these dimensions in a population of rats.

\section{MATERIALS AND METHODS}

Twenty young adult (10 male and 10 female, 10-11 weeks old, 169-435 g) Sprague-Dawley rats were obtained from the University of Newcastle Animal Services Breeding unit and used according to the Australian Code of Practice for the Care and Use of Animals for Scientific Purposes. After death by asphyxiation (100\% $\mathrm{CO}_{2}$ ), the cervical (C1-T1) vertebral column of each rat was dissected and perivertebral muscle tissue removed by gross dissection. The isolated vertebral columns were then placed on an ant nest for 10 days to remove tissue in the vertebral canal. One female vertebral column was lost during this period. The remaining $(n=19)$ vertebral columns were then placed in a $2 \mathrm{M}$ sodium chloride solution and heated to $70^{\circ} \mathrm{C}$ for 15 min to digest the remnant connective tissue. The vertebral column of each rat was disarticulated and each segment identified by the attachment of a small marked suture. This study was performed in two parts. Part 1 involved the determination of the precision of measurement using three different measurement protocols, and part 2 involved the measurement and analysis of the vertebral canal dimensions from the population of rats $(n=19)$.

\section{Precision of Measurement}

In part 1 of this study, the accuracy of measures of the anterior posterior and transverse dimensions of the vertebral canal in the cervical vertebral column of the rat was undertaken. Each of two investigators used each of three methods to determine these dimensions for all vertebra in the cervical vertebral column and the first thoracic vertebra (C1-T1) of one randomly selected rat. In method one, the investigators used a digital caliper (Mitutoyo) to measure the maximum anterior to posterior and transverse dimensions orthogonal to each other as described by Tatarek (2005) and shown in Figure 1. In contrast to Tatarek's (2005) method, we made two measurements for each dimension by placing the calipers into the vertebral canal from the rostral to caudal direction and then from the caudal to rostral direction. The second method involved the use of a camera lucida drawing arm mounted on a dissecting microscope (Zeiss Stem1 SV11) to draw (trace) the perimeters of the vertebral canal, viewed from the rostral to caudal and then caudal to rostral, and a scale bar $(\mathrm{mm})$ on a piece of paper. As in method 1, the investigator then measured the maximum anterior to posterior and transverse dimensions of the vertebral canal on the drawing using the same digital caliper as used in method 1 . However, in method 2, a clear sheet of acetate with two lines drawn orthogonal to each other was placed over the drawings of the vertebral canals to assist in making the anterior to posterior and transverse measurements orthogonal to each other. In the final method each vertebra and a 


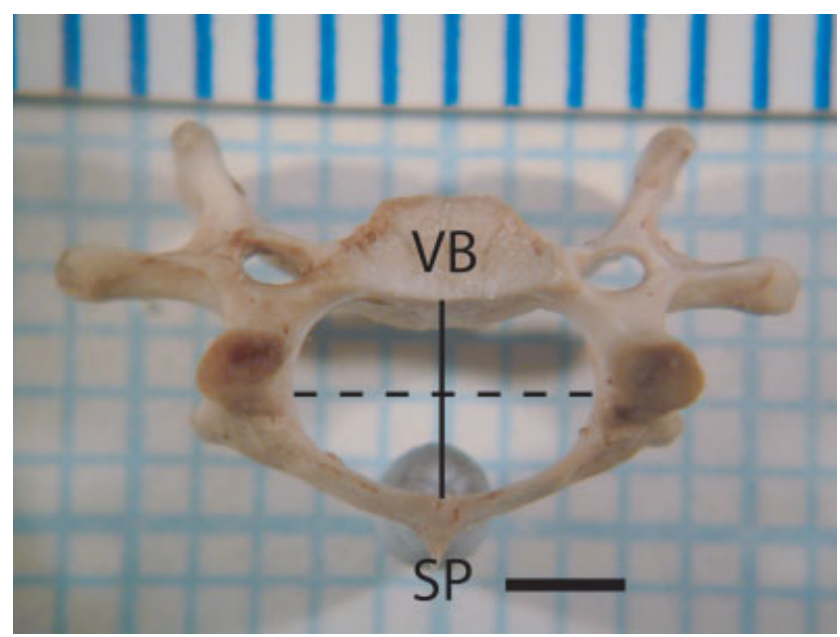

Fig. 1. Figure 1 shows a photomicrograph of the sixth cervical vertebral segment of the rat viewed from rostral to caudal. The anterior to posterior (solid line) dimension between the vertebral body (VB) and the spinous process (SP) and transverse (dashed line) dimensions from left to right have been drawn on the photomicrograph. Scale bar $=2 \mathrm{~mm}$.

scale bar (mm) were simultaneously photographed (Digital Camera image; Nikon Cool Pix 950) with the aid of a dissecting microscope (Zeiss Stem1 SV11) as shown in Figure 1. The digital images of each vertebra were taken in the horizontal plane from rostral to caudal and then from caudal to rostral with the image focused on the rostral or caudal surface, respectively, of the vertebra. All digital images were stored for latter analysis. The investigator then used a computer-based measuring system (ImageJ, US National Institutes of Health, Bethesda; Abramoff et al., 2004) to measure the anterior to posterior and transverse dimensions of the vertebral canal visualized on the digital images of the vertebra.

The investigators used only one method to obtain the dimensions of the vertebral canal on any day and each method of measurement was performed $24 \mathrm{hr}$ after the use of the previous method. The second investigator was blinded to the results of the first investigator and performed the procedures on separate days.

We determined the difference in measurements and the repeatability of the measurements for each of the three methods using data from the one rat as reported above by using the procedures described by Bland and Altman (1986) and in the text by Bland (1987). The data sets from investigators 1 and 2 were first tested for normality using the Kolmogorov-Smirnov test. The coefficient of variation (CV) of the difference between the measures obtained by each of the two investigators for the respective method was determined. Because the data sets were not normally distributed, significant differences between the methods as performed by one investigator was tested by undertaking a Friedman Repeated Measures Analysis of Variance and a post hoc analysis was performed using an All Pairwise Multiple Comparison Procedure (Tukey Test). These procedures were undertaken with the aid of a desktop computer and a commercial statistical software program (SigmaStat V2; SPSS, Inc.).

\section{Vertebral Canal Dimensions}

In part 2 of the study, the vertebral canal dimensions for each vertebra of the 19 cervical and upper thoracic vertebral columns (C1-T2) were determined by the same investigator using the digital photographic images of each vertebra and a computer-based measurement system (ImageJ, US National Institutes of Health, Bethesda). Each vertebral canal was photographed from the caudal and the rostral surface as described above. Data were tabulated, and a descriptive analysis was performed. The $t$-test was used to determine whether there were significant differences between the vertebral canal dimension for each segment obtained from the rostral to caudal and caudal to rostral images of the vertebral canal. Differences between male and female dimensions were tested with Kruskal-Wallis one-way analysis of variance on ranks. Statistical significance for all tests was set at $P<0.05$.

\section{Precision of Measurement}

The CV for the repeat measures (investigator 1 vs. investigator 2 ) on the same rat for method 1 ranged from $4 \%$ (anterior to posterior, rostral surface with mean \pm $\mathrm{SD}$ of the differences of $0.01 \pm 0.14 \mathrm{~mm}$ ) to $8 \%$ (transverse, rostral, and caudal surface with mean $\pm \mathrm{SD}$ of the differences of $0.34 \pm 0.14 \mathrm{~mm})$. Method 2 had a CV ranging from $1 \%$ (transverse, rostral surface, and caudal surface with mean \pm SD of the differences of $0.06 \pm$ $0.14 \mathrm{~mm}$ ) to $5 \%$ (anterior to posterior, caudal surface with mean $\pm \mathrm{SD}$ of the differences of $0.01 \pm 0.17 \mathrm{~mm}$ ), and the CV for method 3 ranged from $2.6 \%$ (anterior to posterior, rostral surface with mean $\pm \mathrm{SD}$ of the differences of $0.02 \pm 0.09 \mathrm{~mm}$ ) to $5.4 \%$ (transverse, rostral surface with mean \pm SD of the differences of $0.28 \pm$ $0.27 \mathrm{~mm}$ ).

There was no apparent correlation between the differences and the mean for method 1, in which the calipers were used to directly measure the specimen, and method 2 in which a drawing of the vertebral canal was made and then the calipers were used to measure the dimensions on the drawing by investigator 1 (Fig. 2A). The mean $( \pm \mathrm{SD})$ of the difference was $0.03 \pm 0.15 \mathrm{~mm}$ and the CV $( \pm \mathrm{SD})$ for these two methods was $3.8 \pm 1.5 \%$. There was no statistically significant difference between these two methods (Fig. 3). Method 3, which involved the computer-based measurement of the digital images of the vertebral canal, generally gave larger results than method 1 and larger differences as the diameter increased (Fig. 2B). The mean of the differences for methods 1 and 3 was $-0.18 \pm 0.23 \mathrm{~mm}$, and the CV was $5.7 \pm$ $0.23 \%$. As indicated in Figure 3, these two methods were significantly different (Tukey Test; $P<0.05$ ). The mean of the differences between method 2 and 3 was $-2.22 \pm$ $0.20 \mathrm{~mm}$, and the CV was $4.9 \pm 0.20 \%$; these two methods gave statistically significant different (Tukey's test $P<0.05$ ) results (Fig. 3). The differences appeared to grow larger as the diameter increased, and method 3 generally gave larger results than method 2 (Fig. 2C).

\section{Vertebral Canal Dimensions}

The mean weight of the rats in part 2 of the study was $261.75 \mathrm{~g}$ (95\% confidence interval [CI], 230.42-293.08 

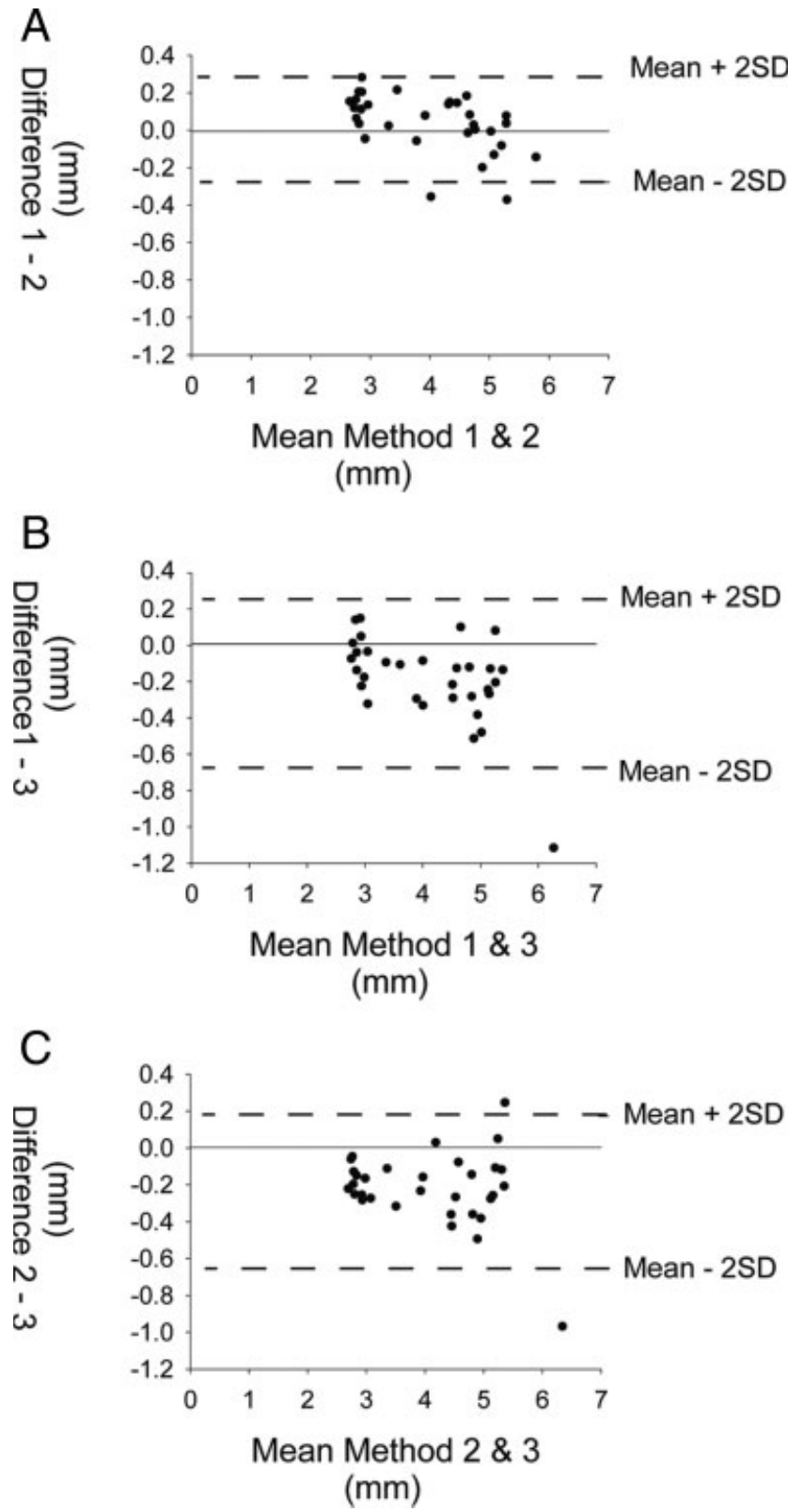

Fig. 2. A-C: The panels show scatter plots of the difference between the two methods ( $y$ axis) and the mean of the two methods ( $x$ axis) for each of the methods used in part 1 of this study (A, methods 1 \& 2; $B$, methods $1 \& 3$; and $C$, methods $2 \& 3$ ). The limits of agreement (mean $\pm 2 \mathrm{SD}$ ) for each scatter plot have been drawn (dashed lines). This method clearly shows that methods 1 and 2 (B and $\mathrm{C}$, respectively) resulted in smaller measurements than method 3 .

g). There was a statistical difference (Student's $t$-test; $P$ $=0.001)$ between the weight of the male $(\mathrm{n}=10$; mean $307.00 \mathrm{~g} ; 95 \% \mathrm{CI}, 259.97-354.03 \mathrm{~g})$ and female $(\mathrm{n}=9$; mean 212.33 g; 95\% CI, 197.37-236.95 g) rats. Table 1 summarizes the anterior to posterior and the transverse dimensions of the vertebral canal for the population of rats in this study. Figure $4 \mathrm{~A}$ shows that the anterior to posterior dimensions of the vertebral canal in the rat are greatest at the upper cervical (C1-C2) level and that

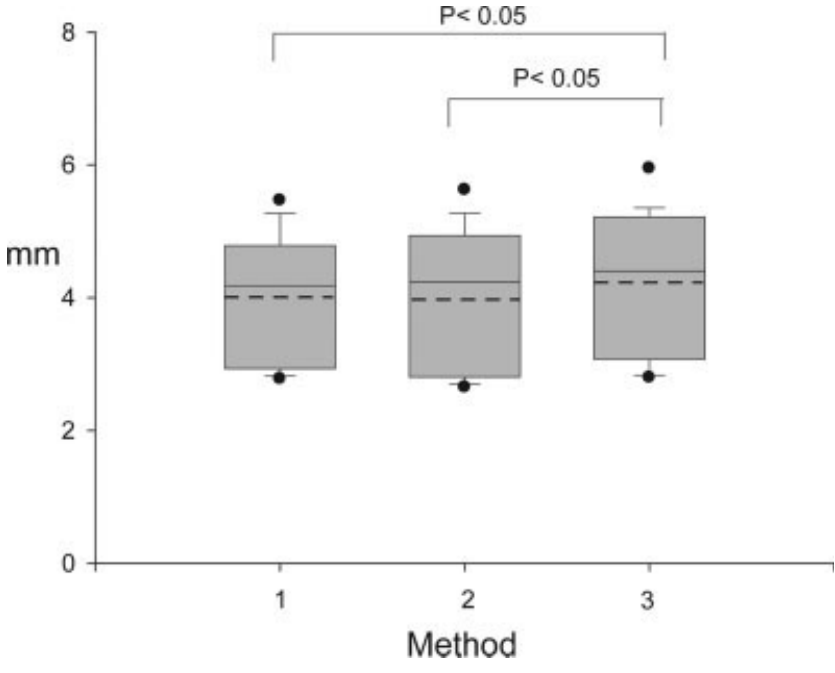

Fig. 3. This figure shows a box plot of the $5 \%$ and $95 \%$ percentiles the 25 to 75 th percentiles (gray box), and the mean (solid line) and median (dashed line) values of the dimensions of the vertebral column (anterior to posterior and transverse data have been combined) of the same rat using three different methods. Methods 1 and 3 and methods 2 and 3 are significantly different $(P<0.05)$.

TABLE 1. Descriptive ${ }^{a}$ data of the anterior to posterior (top panel) and transverse (bottom panel) dimensions of the vertebral column for each vertebral segment (vertebral level C1-T1) in the neck of young adult rats $(n=19)$

\begin{tabular}{lcccc}
\hline $\begin{array}{c}\text { Vertebral } \\
\text { Level }\end{array}$ & $\begin{array}{c}\text { Mean } \\
(\mathrm{mm})\end{array}$ & $\begin{array}{c}\text { SD } \\
(\mathrm{mm})\end{array}$ & $\begin{array}{c}\text { Max } \\
(\mathrm{mm})\end{array}$ & $\begin{array}{c}\text { Min } \\
(\mathrm{mm})\end{array}$ \\
\hline C1 & 5.10 & 0.31 & 5.92 & 4.46 \\
C2 & 3.62 & 0.31 & 4.44 & 2.99 \\
C3 & 3.18 & 0.17 & 3.54 & 2.69 \\
C4 & 3.03 & 0.16 & 3.37 & 2.69 \\
C5 & 2.97 & 0.13 & 3.26 & 2.69 \\
C6 & 2.81 & 0.12 & 3.05 & 2.58 \\
C7 & 2.73 & 0.16 & 3.09 & 2.36 \\
T1 & 2.59 & 0.13 & 2.96 & 2.35 \\
C1 & 6.07 & 0.71 & 7.24 & 4.96 \\
C2 & 4.13 & 0.27 & 4.65 & 3.37 \\
C3 & 4.50 & 0.23 & 4.93 & 4.10 \\
C4 & 4.80 & 0.18 & 5.10 & 4.39 \\
C5 & 4.96 & 0.18 & 5.38 & 4.51 \\
C6 & 5.13 & 0.22 & 5.59 & 4.67 \\
C7 & 5.17 & 0.19 & 5.52 & 4.71 \\
T1 & 4.78 & 0.20 & 5.24 & 4.46 \\
\hline
\end{tabular}

${ }^{a}$ Data presented as mean, standard deviation (SD), and range from maximum ( $\max$ ) to minimum (min) in millimeters.

it progressively decreases in the more caudal segments (C3-T1). The transverse dimension is greatest at the atlas (C1) vertebra and smallest at the level of the axis (C2) vertebra, with a steady increase in the transverse dimension of the more caudal segments with a maximum transverse dimension at the level of the $\mathrm{C} 6$ and $\mathrm{C} 7$ vertebra (Fig. 4B).

There was a significant difference (Tukey's test; $P=$ 0.027 ) in the anterior to posterior dimensions of the $\mathrm{C} 1 \mathrm{ver}-$ terba of male $(n=10)$ and female $(n=9)$ rats. However, 

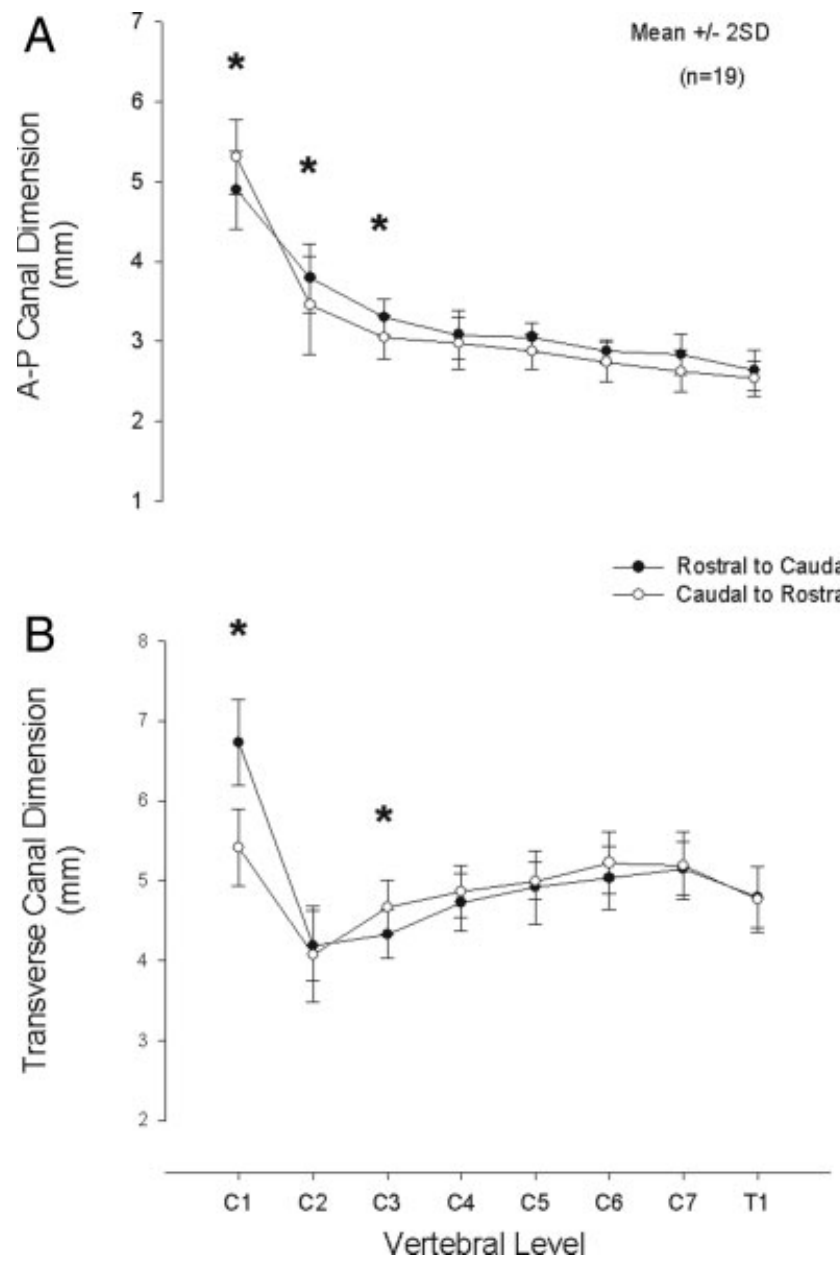

Fig. 4. A,B: This figure is a scatter plot of the mean $( \pm 2 \mathrm{SD})$ of the anterior to posterior $(A)$ and transverse $(B)$ dimensions for each vertebral segment for $\mathrm{C} 1$ to $\mathrm{T} 1$. The rostral surface measurement (closed circles) and caudal surface measurement (open circles) data for each vertebral level have been plotted. Those segments in which there was a significant differences $(P<0.05)$ between the rostral and caudal measurements have been identified with an asterisk.

there was no significant difference in the transverse dimensions of $\mathrm{C} 1$ or in either of the anterior to posterior or transverse dimensions at other vertebral levels of the male and female rats.

The CV for differences between rostral surface and caudal surface measurements for $\mathrm{C} 1, \mathrm{C} 2$, and $\mathrm{C} 3$ vertebra were greater than 5\%, indicating that the differences were not just due to variability in the measurement method (cf. part 1, above). There was a statistical difference $(P<0.001)$ between the rostral surface and caudal surface measurements of $\mathrm{C} 1, \mathrm{C} 2$, and $\mathrm{C} 3$ in the anterior to posterior dimension. Similarly, there were significant differences between the rostral surface and caudal surface measurements of $\mathrm{C} 1$ and $\mathrm{C} 3$ vertebra in the transverse dimension.

Figure 5 shows a comparison of the anterior to posterior and the transverse dimensions of the vertebral column of the rat, based on the data obtained in this study and the human data reported in a study by Tatarek (2005). It can be seen in Figure 5A that the anterior to
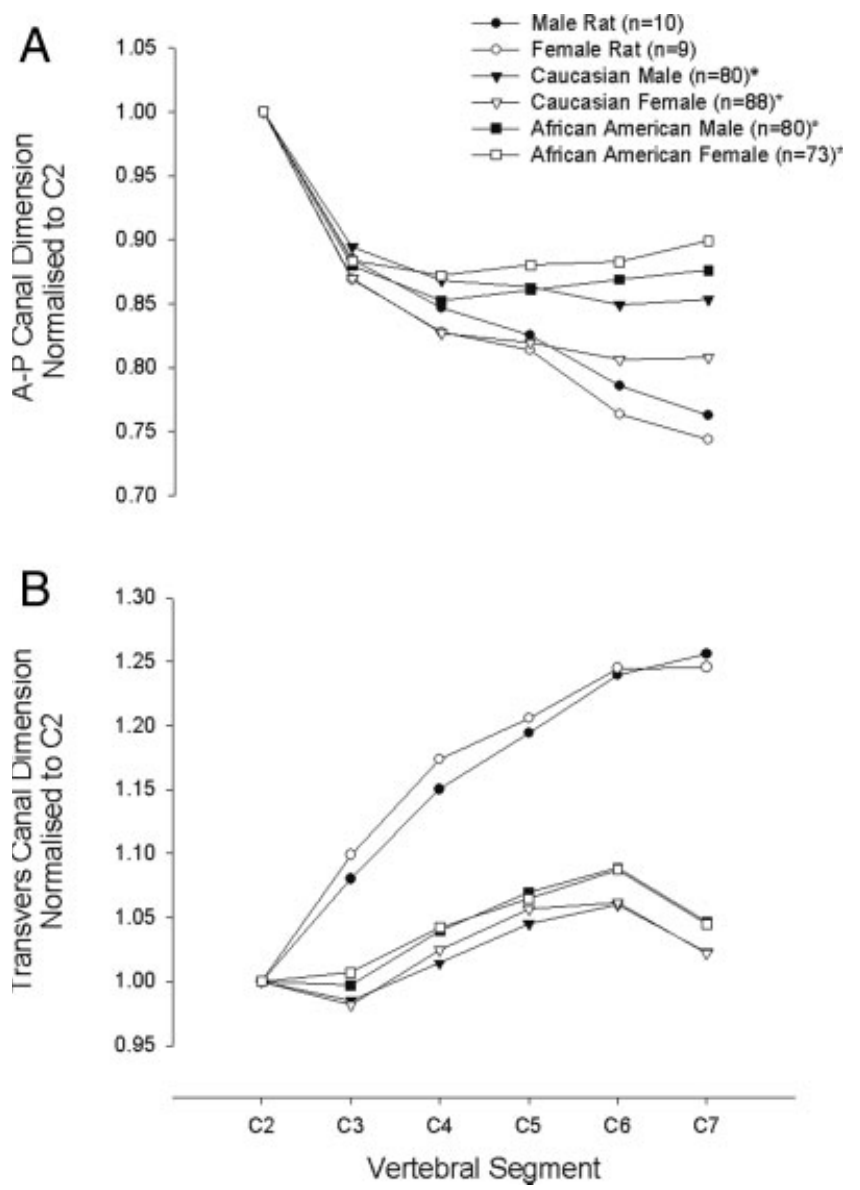

Fig. 5. A,B: This figure is a scatter plot of the mean anterior to posterior (A) and transverse (B) dimensions normalized to the respective dimension of the $\mathrm{C} 2$ vertebral segment. The rat data are from this study, and the human data have been derived from the study reported by Tatarek (2005).

posterior dimension of the rat vertebral canal continues to diminish with each caudal (C3-C7) segment from C2 while in the human this dimension remains relatively constant in the more caudal (C3-C7) segments. The transverse dimension of the rat cervical vertebral canal increases with each caudal (C3-C7) vertebral segment. In contrast, the transverse dimension of the human vertebral canal increases caudally $(\mathrm{C} 4-\mathrm{C} 6)$ and then reduces at the level of the $\mathrm{C} 7$ vertebral segment (Fig. 5B).

\section{DISCUSSION}

Previous ex vivo measurement of the skeletal elements of the cervical vertebral column has involved a variety of methods and equipment including the use of Vernier calipers, micrometers, morphometer, cryomicrotome sections, and digitized (video camera) images of postmortem specimens, whereas in vivo measurements have used plain film X-ray and computed tomography images of the vertebral column (Herzog et al., 1991; Panjabi et al., 1993; Pettersson et al., 1995; Wilke et al., 1997; Grave et al., 1999; Johnson et al., 1999; Yoganandan et al., 2003; Lim and Wong, 2004; Tatarek, 2005). 
The measurement error has only been reported for a few of these devices. Panjabi et al. (1991) report that the error in using their purpose-built morphometer on human specimens was $<5 \%$, whereas Grave et al. (1999) report the error in tracing and digitizing plain film Xrays of humans from repeat data sets ranged from -0.25 to $0.90 \mathrm{~mm}$. Part 1 of the study reported here indicates that the use of Vernier calipers to measure the vertebra (method 1) or camera lucida drawings of the vertebra (method 2) or the use of a computer-based measurement of digitized images of the vertebral segments of the rat's neck (method 3) result in measurement errors that, although slightly larger in some instances (range, 1-8\%) are of the same order of magnitude as those reported in the studies of human vertebra $(<5 \%)$. Our data clearly show that the use of Vernier calipers (method 1) to directly measure the dimensions of the vertebral canal in the rat introduces a higher potential error due to investigator-associated measurement differences than the use of the Vernier calipers to measure drawings or computer-based measurement of digital images of the same vertebra. Although previous data on postmortem specimens of human vertebra were obtained by directly measuring vertebra with Vernier calipers, we chose to use digital images of the vertebra with a computer-based measurement system (method 3 ) in our population study for several reasons. First, the use of Vernier calipers in our study of the rat by either directly measuring the vertebra (method 1) or measuring camera lucida drawings of the vertebra (method 2) were found to have greater measurement error than method 3. Second, method 2 had measurement errors that were not significantly different to method 1 . Third, method 3 gave larger values for the canal dimensions than the other techniques (method 1 and 2) performed in this study and, thus, allowed us to detect small differences in vertebral canal dimensions from one vertebra to another.

Although the rats in our study were only young (11week-old) adults (sexually mature with expected life span 2-3.5 years; Poole, 1987; Pass and Freeth, 1993), there was a significant difference in the weights of the male and female rats. Interestingly, there was no significant difference in the vertebral canal dimensions between the male and females. The spinal cord dimensions in the rat have only been derived from male rats (Portiansky et al., 2004); therefore, no comparison can be made between the spinal cord and vertebral canal dimensions in the rat. In contrast, post hoc analyses $(t$ test, assuming normal distribution) of the human data reported by Tatarek (2005; cf Table 1) indicate that the vertebral canal dimensions are larger in the human male than female. The sexual dimorphism of the cervical vertebra has been clearly identified (Grave et al., 1999). Despite these differences in the vertebral canal dimensions in the human, there are no differences in the anterior to posterior or transverse dimensions of the spinal cord based on sex or age of adults (Kostas et al., 1998). It remains to be determined if older rats (e.g., 50-80 week old) or aged rats (100-150 week old) or rats of a different strain have vertebral canal dimensions in the neck that significantly differ from those reported here. This is the focus of ongoing work in our laboratory. There is evidence that bipedism and upright posture can change vertebral canal dimensions in the rat lumbar vertebra (Cassidy et al., 1988).
Our rat data are consistent with that of the human and sheep in some respects (Panjabi et al., 1991; Wilke et al., 1997; Tatarek, 2005). As reported by Kida et al. (1999), the vertebral canal of the young adult rat has its largest anterior to posterior dimension in the upper cervical (C1-C2) vertebral segments. Furthermore, with the exception of the atlas vertebra $(\mathrm{C} 1)$, the transverse dimension of the vertebral canal in the neck is larger in the lower cervical and upper thoracic (C5-T1) vertebral column. We noted significant differences in the measurements of the vertebral canal in the rat if measured from the rostral surface compared with the caudal surface in the upper (C1-C3) but not the lower cervical and upper thoracic (C4-T1) vertebra. This finding suggests that the vertebral canal in these segments has a different rostrocaudal conformation than the lower cervical vertebra in the rat. However, this difference was not formally tested in the current study.

In contrast to the human data (Tatarek, 2005), our data show that the anterior to posterior dimension of the vertebral canal in the neck of the rat is reduced in size in each successively more caudal vertebral segment in the neck (C1-T1). This finding has also been reported in the sheep (Wilke et al., 1997). The transverse dimension of the vertebral canal in the rat increased with each segment caudal to the axis (C2) vertebra, which is consistent with data from the human, although the human vertebral canal reduces its transverse dimension at the level of C7 (Panjabi et al., 1991; Tatarek, 2005), whereas our data indicates that the rat, like the sheep (Wilke et al., 1997), does not.

A study by Portiansky et al. (2004) has shown that the cross-sectional area of the cervical spinal cord in the rat is largest in the upper cervical region (C1-3) but abruptly reduces to approximately $64 \%$ of that of the upper cervical cord in the lower cervical spinal cord segments (C4-C8) and that the rat does not have a cervicothoracic enlargement. In contrast, the human has a well-defined enlargement in the cervicothoracic region of the spinal cord at the level of C4-C6 (Sherman et al., 1990). These species differences in the dimensions of the vertebral canal of the cervical vertebra may simply be a reflection of the differences in the dimensions of the spinal cord between species. However, it is noteworthy that the upright biped human, typically, has a very mild lordosis at the cervicothoracic junction of the vertebral column, whereas quadruped animals such as the rat have a severe lordosis at the cervicothoracic region of the vertebral column (Vidal et al., 1986; Kumar et al., 2000). This difference may also account, in part, for the differences in the dimensions of the vertebral canal in the neck of quadrupeds and bipeds including the human.

\section{ACKNOWLEDGMENTS}

This project was funded by the Australian Spinal Research Foundation, and J.R.F. received a Summer Scholarship by the School of Biomedical Sciences at the University of Newcastle.

\section{LITERATURE CITED}

Abramoff MD, Magelhaes PJ, Ram SJ. 2004. Image processing with ImageJ. Biophoton Int 11:36-42.

Bland JM. 1987. An introduction to medical statistics. Oxford, UK: Oxford Medical Publications. p 276-282. 
Bland JM, Altman DG. 1986. Statistical methods for assessing agreement between two methods of clinical measurement. Lancet 1:307-310.

Bolton PS, Budgell B, Kimpton A. 2006. Influence of innocuous cervical vertebral movement on the efferent innervation of the adrenal gland in the rat. Auton Neurosci 124:103-111.

Cassidy JD, Yong-Hing K, Kirkaldy-Willis WH, Wilkinson AA. 1988 A study of the effects of bipedism and upright posture on the lumbosacral spine and paraverterbal muscles of the wistar rat. Spine 13:301-308.

Fiford RJ, Bilston LE, Waite P, Lu J. 2004. A vertebral dislocation model of spinal cord injury in rats. J Neurotrauma 21:451458.

Graf W, De Waele C, Vidal PP. 1995. Functional anatomy of the head-neck movement system of the quadrupedal and bipedal mammals. J Anat 186:55-74.

Grave B, Brown T, Townsend G. 1999. Comparison of cervicovertebral dimensions in Australian Aborigines and Caucasians. Eur J Orthodont 21:127-135.

Grill RJ. 2005. User-defined variables that affect outcome in spinal cord contusion/compression models. Exp Neurol 196:1-5.

Herzog RJ, Wiens JJ, Dillingham MF, Sontag MJ. 1991. Normal cervical spine morphometry and cervical spinal stenosis in asymptomatic professional football players. Spine 16:178-186.

Johnson DR, McAndrew TJ, Oguz O. 1999. Shape differences in the cervical and upper thoracic vertebrae in rats (Rattus norvegicus) and bats (Pteropus poicephalus): can we see shape patterns derived from position in column and species membership? J Anat 194:249-253.

Kida MY, Johnson DR, McAndrew TJ, O’Higgins P. 1999. Adaptation in the vertebral column: a comparative study of patterns of metameric variation in seven species of small mammals. J Anat 194:207-214.

Kostas F, Kapsalaki E, Jackson J, Vogel RL, Robinson JS. 1998. Cervical spinal cord-smaller than considered? Spine 23:15131516.

Kumar N, Kukreti S, Ishaque M, Mulholland R. 2000. Anatomy of deer spine and its comparison to the human spine. Anat Rec 260:189-203.
Lee KE, Thinnes JH, Gokhin DS, Winkelstein BA. 2004. A novel rodent neck pain model of facet-mediated behavioral hypersensitivity: implications for persistent pain and whiplash injury. J Neurosci Methods 137:151-159.

Lim J-K, Wong H-K. 2004. Variation of the cervical spinal Torg ratio with gender and ethnicity. Spine 4:396-401.

Panjabi M, Duranceau J, Groe V, Oxland T, Takata K. 1991. Cervical human vertebra. Quantitative three-dimensional anatomy of the middle and lower regions. Spine 16:861-869.

Panjabi MM, Oxland T, Takata K, Goel V, Duranceu J, Krag M. 1993. Articular facets of the human spine. Spine 18:1298-1310.

Pass D, Freeth G. 1993. The rat. ANZCART News (insert) 6:1-4.

Paxinos G. 2004. The rat nervous system. 3rd ed. San Diego: Elsevier Academic Press.

Pettersson K, Karrholm J, Toolanen G, Hildingsson C. 1995. Decrease width of the spinal canal in patients with chronic symptoms after whiplash injury. Spine 20:1664-1667.

Portiansky EL, Barbeito CG, Goya RG, Gimeno EJ, Zuccolilli GO. 2004. Morphometry of cervical segments grey matter in the male rat spinal cord. J Neurosci Methods 139:217-229.

Poole TB. 1987. The UFAW Handbook on the care and management of laboratory animals. 6th ed. Essex: Longman Scientific and Technical. p 321-323.

Sato A, Swenson RS. 1984. Sympathetic nervous system response to mechanical stress of the spinal column in rats. J Manipulative Physiol Ther 7:141-147.

Sherman JL, Nassaux PY, Citrin CM. 1990. Measurements of the normal cervical spinal cord on MR imaging. Am J Neuroradiol 11:369-372.

Tatarek NE. 2005. Variation in the human cervical neural canal. Spine 5:623-631.

Vidal PP, Graf W, Berthoz A. 1986. The orientation of the cervical vertebral column in unrestrained awake animals. I. Resting position. Exp Brain Res 61:549-559.

Wilke H-J, Kettler A, Wenger KH, Claes LE. 1997. Anatomy of the sheep spine and its comparison to the human. Anat Rec 247:542-555.

Yoganandan N, Knowles SA, Maiman DJ, Pintar FA. 2003. Anatomical study of the morphology of human cervical facet joint. Spine 28:2317-2323. 